\title{
Dynamics of interval IUD use in India
}

The Evidence Project

Follow this and additional works at: https://knowledgecommons.popcouncil.org/departments_sbsr-rh

Part of the Demography, Population, and Ecology Commons, Family, Life Course, and Society Commons, International Public Health Commons, Maternal and Child Health Commons, and the Women's Health Commons How does access to this work benefit you? Let us know!

\section{Recommended Citation}

The Evidence Project. 2018. "Dynamics of interval IUD use in India: Quality of care received and interim results three months after insertion," research brief. Washington, DC: Population Council, The Evidence Project. 


\section{Dynamics of Interval \\ IUD Use in India}

\section{Quality of care received and interim results} three months after insertion

\section{KEY RECOMMENDATIONS}

\section{Disseminate messaging about potential side effects of the IUD}

Nearly 30 percent of those who discontinued using the intrauterine device (IUD) did so due to side effects or other health concerns. In addition, less than half of IUD users were told about the potential side effects, what to do if they experience side effects, or the warning signs of the method. Providers and accredited social health activists (ASHA) should inform women of potential side effects and alleviate any concerns related to fertility. Furthermore, social behavioral change programs may consider including specific messaging on common side effects of the IUD.

\section{Institutionalize quality of care in pre-service trainings of all family planning providers and frontline health workers}

To improve quality of care during family planning visits for all contraceptive methods, providers and frontline health workers should receive training on all four domains of quality of care: 1) respectful care, 2) counseling to enable appropriate method selection, 3) counseling on effective use of method chosen, and 4) information on continuation of contraceptive use and care.

\section{Train frontline health workers to visit and provide counseling to recent adopters of the IUD}

Frontline health workers have a critical opportunity to provide information to recent adopters of the interval IUD who are dissatisfied with the method. While most users had spoken to frontline health workers about the IUD before starting the method, less than half of those who discontinued the IUD spoke to an ASHA about their desire to stop. ASHAs and other frontline health workers should be trained to visit all women who recently began using the interval IUD to understand their concerns and fertility desires and counsel those who want to stop using the IUD but continue preventing pregnancy to voluntarily switch to another suitable method.

\section{Engage husbands of recent interval IUD adopters}

Most women who discontinued the interval IUD spoke to their husband about their desire to stop using the method. The advice given by most of these husbands was to stop using the method, and only 13 percent suggested switching to another method. As women are going to their husbands for support, husbands should be equipped to encourage their wives to consider other modern methods as long as their desire to prevent pregnancy remains. 


\section{INDIA CONTRACEPTIVE USE DYNAMICS LONGITUDINAL STUDY METHODS}

Through the Evidence Project, Population Council researchers are conducting a longitudinal study of 2,699 married women aged 15-49 who began using IUD/PPIUD, injectable, or oral contraceptive pill (OCP). These reversible contraceptive users are first being interviewed within one month of starting the method (known as the enrollment survey) and at 3-, 6- and 12-months follow-up. Respondents from Haryana and Odisha states in India were enrolled into the study from December 2016 to October 2017 (see Map 1). In Haryana, all respondents were recruited through accredited social health activists (ASHAs) at the community level. In Odisha, PPIUD users were recruited at government health facilities, interval IUD and OCP users were recruited primarily through ASHAs, and injectable users were recruited primarily at non-governmental organization (NGO) facilities.

The purpose of this research study is to provide evidence that can be used to strengthen the family planning program and meet the needs of reversible contraceptive users who want to prevent pregnancy in India. With support from USAID/India, Population Council researchers are exploring contraceptive use dynamics of married women by conducting a cohort study with the following research objectives:

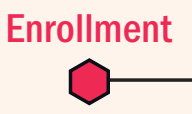

Month 3

Month 6

\section{BACKGROUND}

As articulated in its FP2020 commitment, India is looking to expand its method mix to include a greater reliance on modern spacing methods, including the long-acting and reversible IUD (MOHFW, 2014). Reliance on female sterilization in India is high: 36 percent of currently married women use sterilization (IIPS and ICF, 2017a) accounting for 75 percent of modern contraceptive use. As female sterilization is permanent, and regret for undergoing sterilization has been reported by seven percent of sterilized women (IIPS and ICF, 2017b), the IUD is a potential alternative for women who may be unsure if they want to limit childbearing. However, IUD use (both postpartum and interval) among currently married women remains low in India, at 1.5 percent (IIPS and ICF, 2017a), despite being offered free of charge through India's National Family Planning Program (MOHFW, 2013). This is due in part to a lack of up-to-date information about the IUD among health
1. To assess one-year modern spacing contraceptive discontinuation rates by modern spacing methods (postpartum IUD/interval IUD, injectables, OCPs) among a cohort of modern spacing contraceptive users.

2. To measure influencing facilitators for contraceptive continuation and discontinuation, including intensity of experienced side effects.

3. To measure the Method Information Index (MII) that measures client's recall of counseling information received.

4. To assess influMap 1
Study Implementation Sites encing factors that lead to contraceptive switching or non-switching.

5. To explore providers' attitudes about contraceptive discontinuation and switching, and their practices with clients who want to discontinue or switch.

Month 12 providers and potential clients; the disadvantages of the IUD are often exaggerated, and misconceptions are widespread (MOHFW, 2013). Previous research has shown that women and men share misconceptions, including that the IUD causes cancer, weight gain, pain while walking, and pain for men during intercourse. Providers also hold negative beliefs, such as a) IUDs cause infection and sterility and b) will be expelled if a woman has had too many children (Khan et al., 2007). To combat these myths and inform the National Family Planning Program's continued scale-up of the interval IUD (NHM, 2017), more research on the experiences of women currently using the interval IUD is necessary.

The purpose of this brief is to provide evidence that can be used to strengthen the family planning program in India, particularly for the provision of interval IUDs. While data are available for injectable, oral contraceptive pill (OCP), and postpartum IUD (PPIUD) users enrolled in the study (see Box 1), 
this brief focuses on interval IUD users (IUD users who accepted the method at a time other than postpartum) and describes a) their characteristics, b) quality of care received at IUD insertion, c) contact with frontline health workers, and d) contraceptive continuation three months after enrollment. This study did not observe providers in these family planning counseling interactions.

\section{METHODS}

Box 1 presents the methodology of the longitudinal study of reversible contraceptive users in India. Of 2,699 women enrolled in this study, this brief looks at the 640 women who have started a new episode of interval IUD use.

\section{RESULTS}

\section{BACKGROUND CHARACTERISTICS}

The median age of interval IUD users enrolled in the study was 25 years. More than half (54\%) of interval IUD users had attended secondary school or higher, 23 percent had attended primary or middle school, and 23 percent had never attended school (see Figure 1). Ninety-three percent of respondents were Hindus and seven percent were Muslims. Ninety-two percent were housewives, five percent farmed their own land, and one percent were employed as office attendants. Although all respondents were married, eight percent were not living with their husband at the time of the enrollment survey.

\section{Figure 1}

\section{Background characteritics of interval IUD users ( $n=640$ )}
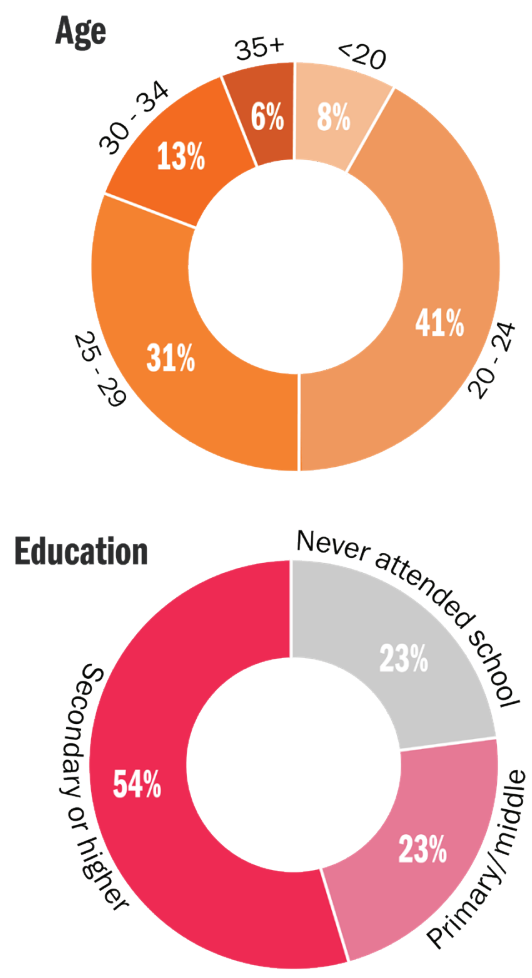

More than half of respondents (63\%) had used a family planning method before. Among those who had previously used a method $(n=406)$, the most recent methods used before the current episode of interval IUD use were condoms (28\%), abstinence $(20 \%)$, OCP (19\%), IUD (16\%), withdrawal (11\%), injectables (2\%), rhythm method (2\%), emergency contraceptives (1\%), lactational amenorrhea method (1\%), and Standard Days Method (0.3\%). Respondents' median age at first contraceptive use was 23 years.

Ninety-nine percent of respondents had at least one living child. Thirty-nine percent had one child, 37 percent had two children, and 24 percent had three or more children. Sixty-two percent of respondents do not want to have any more children in the future. Twenty-seven percent wanted to wait two years before their next child, three percent wanted to wait between one and two years, and one percent wanted a child in the next year. Seven percent were undecided about whether or when to have another child (see Figure 2).

\section{Figure 2}

\section{Percent distribution of interval IUD users by fertility} preferences at enrollment $(n=640)$

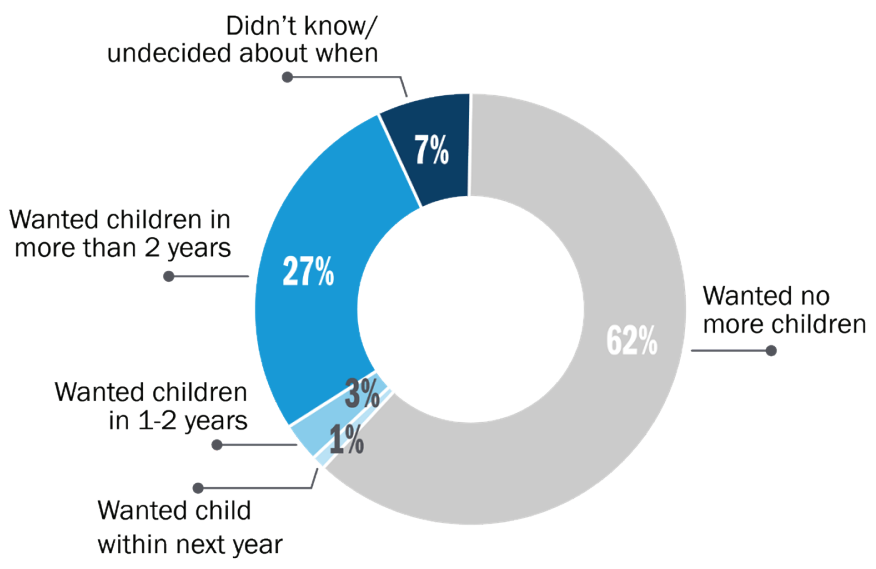

\section{QUALITY OF CARE RECEIVED AT INTERVAL IUD INSERTION}

Seventy-two percent of respondents received their interval IUD from a government hospital. Eighteen percent received the method from a frontline health worker (trained auxiliary nurse midwives can provide IUDs in India; Scott, 2014), six percent from a private facility, and four percent from an NGO. Figure 3 presents 22 items measuring quality of care received during these initial visits. These measures of quality are broken down into four domains of process quality (Jain, Townsend \& Ramarao 2018): 1) respectful 
Box 2

\section{What is quality of care?}

Measures of quality are broken down into four domains of process quality (Jain et al., 2018):

1. Respectful care: Concerns interpersonal interactions between the provider and client and assesses aspects of privacy and confidentiality.

2. Method selection: Questions focus on information that a provider should seek to enable appropriate method selection at the decision-making point.

3. Effective use of the selected method: Information given to the respondent about the method selected.

4. Continuity of contraceptive use and care: Includes follow-up appointments and the ability to change methods.

Figure 3

\section{Aspects of process quality during IUD insertion reported by interval IUD users $(n=640)$}

\section{RESPECTFUL CARE}

Treated well by provider
Questions answered to satisfaction
Respondent allowed to ask questions
Felt information will be kept confidential
Felt audio privacy
Felt visual privacy
METHOD SELECTION

Was asked preferred FP method

Was asked desire for another child

Was told about other methods

Was asked preferred timing of next child

Was asked previous FP experience

Received information about methods that protect against STIs

Received information without any method being promoted

How to use the method

How chosen method works

Side effects of the method

How to manage problems

Warning signs associated with the method CONTINUITY OF CONTRACEPTIVE USE AND CARE

Told about timing of next visit Possibility of switching the method Told about other sources of supply

Given appointment card for follow-up visit care, 2) method selection, 3) effective use of the selected method, and 4) continuity of contraceptive use and care (see Box 2).

\section{Respectful care}

Nearly all respondents (99\%) reported that they were treated well or very well by the provider. The majority of interval IUD users also felt their questions were answered to their satisfaction (86\%) and felt the provider allowed them to ask questions (80\%). While seventy-eight percent felt the information they shared with the provider would be kept confidential, slightly fewer felt they had audio (70\%) and visual privacy $(68 \%)$.

\section{Method selection}

Interval IUD users reported mixed levels of information obtained from providers. Most (73\%) respondents reported that the provider asked about their preferred family planning method. Fewer were told about other methods of family planning (69\%)

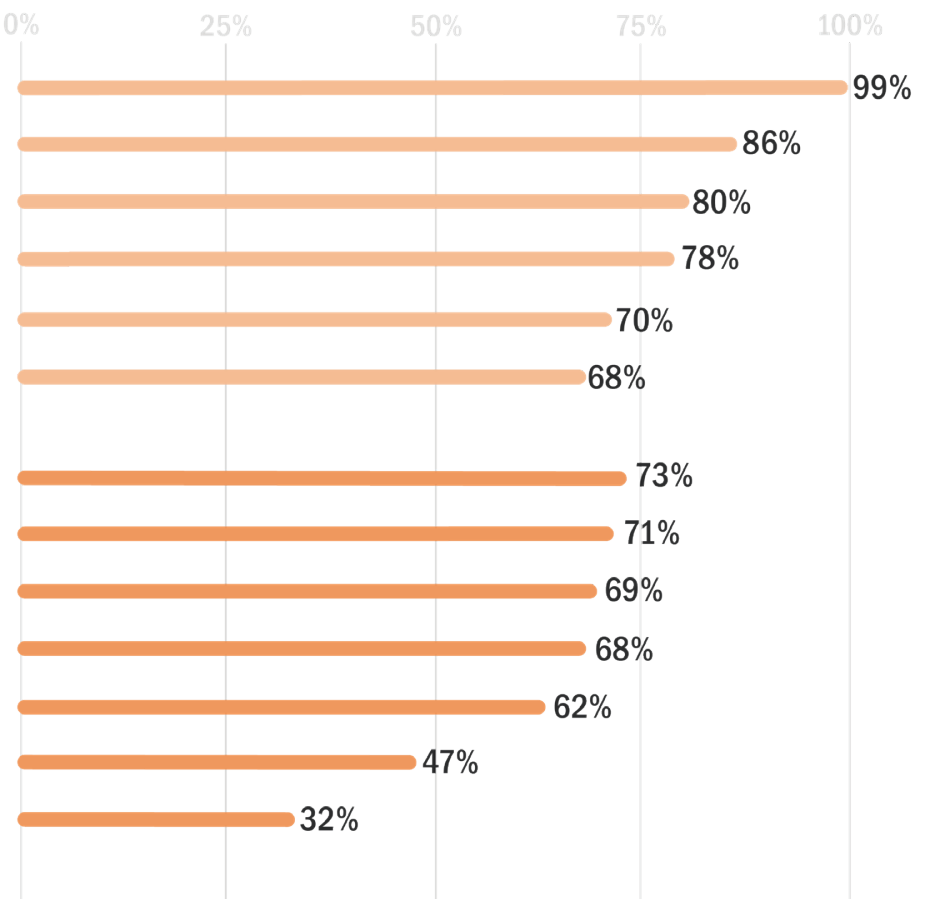




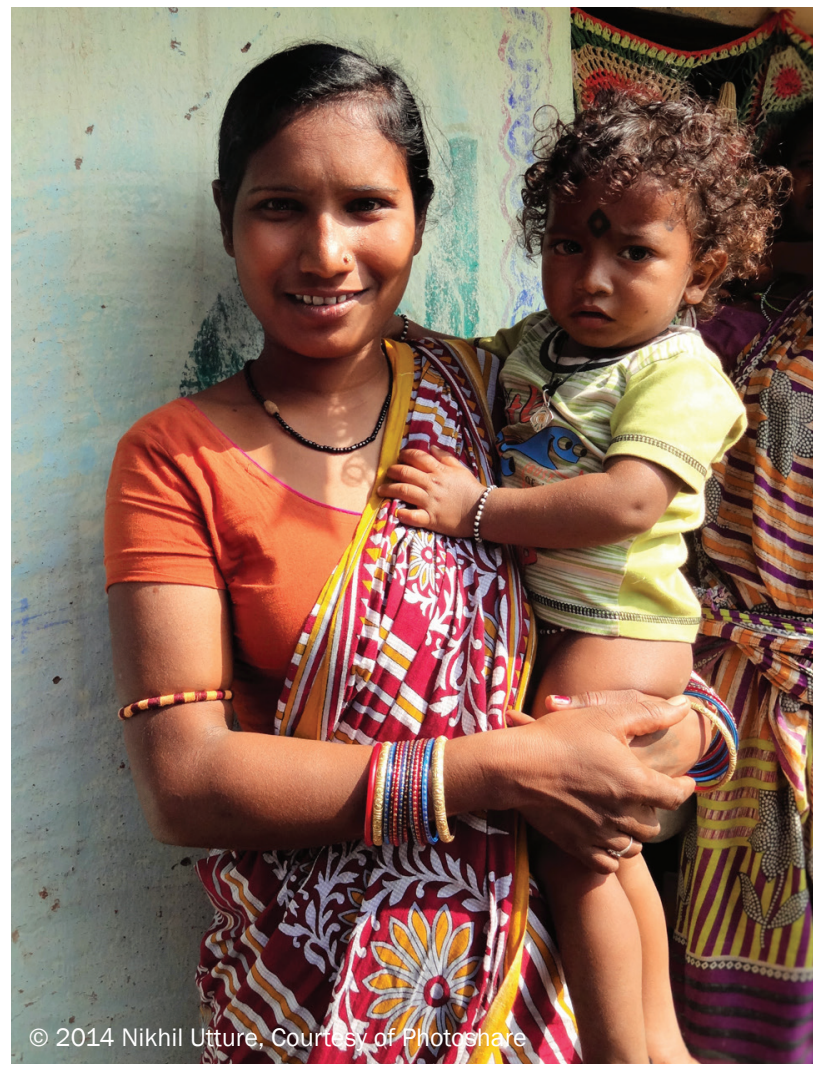

while 62 percent were asked about previous family planning experience. In terms of fertility intentions, seventy-one percent were asked about their desire to have another child and 68 percent were asked about the preferred timing of the next child. Less than half $(47 \%)$, however, were given information about methods that protect against STIs and HIV and only 32 percent felt they had received information without any method being promoted.

\section{Effective use of method selected}

Although most interval IUD users reported that they were told how to use the method (85\%) and how the method works $(79 \%)$, less than half were told about possible side effects of the method (47\%), warning signs associated with the method (45\%), or how to manage possible side effects (45\%). The side effects that interval IUD users reported that providers discussed with them were backache (26\%), irregular menstruation (14\%), nausea (13\%), abdominal pain $(12 \%)$, and vaginal discharge (12\%) (data not shown).

\section{Continuity of contraceptive use and care}

Though eighty-one percent of interval IUD users were told when to return for a follow-up visit, only half (50\%) were given an appointment card for this visit. Seventy-one percent reported being informed about the possibility of switching methods if the IUD is not suitable, and 58 percent reported their provider told them about other sources of supply for family planning methods.

\section{CONTRACEPTIVE METHOD DECISION MAKING}

Respondents were asked who made the final decision about the method they received at enrollment after discussions with the provider (Figure 4). Threequarters of interval IUD users reported being involved in the final decision: 23 percent reported making the final decision by themselves, 42 percent made the decision with their husband, and 10 percent with their health provider. One-quarter reported that someone else made the final decision: 15 percent said their husband made the final decision and 10 percent said their health provider made the final decision. Nearly all (92\%) interval IUD users reported receiving the method that they wanted, although eight percent reported they did not receive the method they wanted or were unsure (data not shown). Reasons why women did not get their preferred method was not collected in this study.

\section{CONTACT WITH FRONTLINE HEALTH WORKERS}

At enrollment, interval IUD users were asked about their contact with frontline health workers. Eightyeight percent of respondents had met with a frontline health worker at some point prior to the study. When asked about what type of frontline health worker provided information on the IUD, 75 percent reported receiving this information from an ASHA, 10 percent from an auxiliary nurse midwife (ANM), and 3 percent from an Anganwadi worker (AWW). Of the 640 interval IUD users, 86 percent had met with a frontline health worker in the three months preceding the survey. Seventeen percent met with a frontline health worker once in the three months preceding the survey, 50 percent met between two

\section{Figure 4 \\ Percent distribution of interval IUD users by who made the final decision about contraceptive method $(n=640)$}

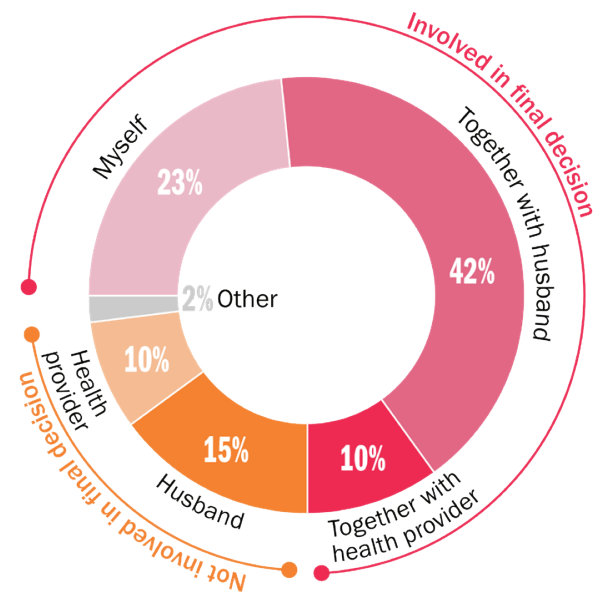


and five times, 20 percent met more than five times, and 14 percent had not met a frontline health worker the three months prior to the survey.

Topics discussed with frontline health workers in the three months preceding the survey are presented in Figure 5. The most common topic discussed was available contraceptive methods (42\%), followed by fertility intentions (23\%) and side effects (20\%). Other topics discussed include long acting methods, how to deal with side effects, and return to fertility. Twenty percent of respondents could not recall what was discussed with frontline health workers.

\section{Figure 5}

Topics discussed with frontline health workers in the three months preceding the survey reported by interval IUD users $(n=640)$

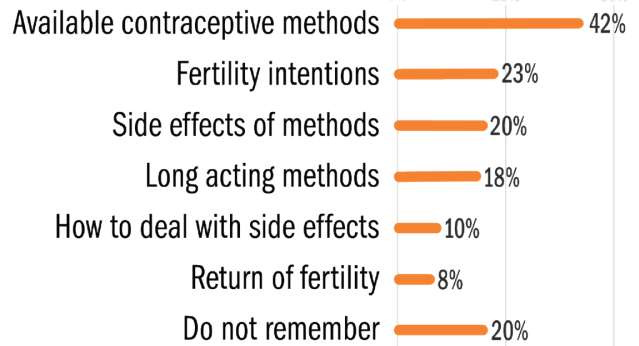

\section{CONTRACEPTIVE USE AT THREE MONTHS FOLLOWING INTERVAL IUD INSERTION}

Eighty-five percent of interval IUD users completed the follow-up survey $(n=545)$. Three months after beginning interval IUD use, 87 percent were still using the IUD. Four percent had switched from the IUD to a different modern method, five percent had switched to a traditional method, and four percent had stopped using contraception altogether. The modern methods switched to after removing the IUD were condoms ( $2 \%$ of IUD users interviewed at three-month), OCP (1\%), injectables (0.6\%) and female sterilization (0.6\%).

Of the 21 women who stopped contraception altogether, eight women reported not wanting to have children in the future, four reported wanting to wait more than two years before having another child and four percent were undecided about the timing of their next child. Only five wanted to have a child within the next two years. Thus, most women who discontinued the interval IUD and did not switch to another method still have an unmet need for family planning.

\section{Reasons for interval IUD discontinuation}

Discontinuers of the interval IUD (both those who discontinued contraception altogether and those who switched to another method, $n=68$ ) provided one or more reasons for stopping use of the IUD (Figure 6). Nine percent discontinued the IUD because they desired to get pregnant. The most common reason listed for discontinuing the IUD was side effects and health concerns (29\%). Less than 1 in 5 women reported that their IUD was expelled (15\%) their husband was not living at home $(12 \%)$ and their husband was opposed (7\%). Other reasons reported were the IUD was inconvenient to use (7\%), method failure/pregnancy (6\%), they were not having sex (6\%), they had not menstruated since starting the method (4\%), the IUD interferes with the body's normal processes (4\%), and wanting to switch to female sterilization (3\%).

\section{Figure 6 \\ Reasons for discontinuation reported by interval IUD discontinuers at three month follow-up $(n=68)$}

\begin{tabular}{|c|c|}
\hline Side effects/health concerns & $15 \%$ \\
\hline IUD expelled & $15 \%$ \\
\hline Husband not living at home & $12 \%$ \\
\hline Wants to become pregnant & $9 \%$ \\
\hline Husband opposed & $-7 \%$ \\
\hline Inconvenient to use & $\rightarrow \%$ \\
\hline Method failure/pregnancy & $-6 \%$ \\
\hline Not having sex & $-6 \%$ \\
\hline Not menstruated since start of method & $-4 \%$ \\
\hline nterferes with body's normal processes & $-4 \%$ \\
\hline Wants to switch to sterilization & $-3 \%$ \\
\hline
\end{tabular}

\section{Discussions about discontinuation}

Respondents were asked to whom they spoke about their desire to discontinue using the interval IUD. Among respondents who discontinued the IUD (switchers and stoppers, n=68), 59 percent spoke with their husband (see Figure 7). Forty-one percent spoke with an ASHA, 21 percent to their mother in-law, and 10 percent to their provider. Fewer spoke to a sister-in-law, a sister, their mother, or someone else (3\% each). Twenty-one percent did not speak to anyone about their desire to stop using the IUD. Respondents who discontinued the IUD $(n=68)$ were also asked if they spoke about stopping the method with the same provider/frontline health worker who gave them the IUD. Fifty-eight percent returned to the same provider or frontline 
Figure 7

\section{Individuals with whom interval IUD discontinuers discussed their desire to discontinue the method $(n=68)$}

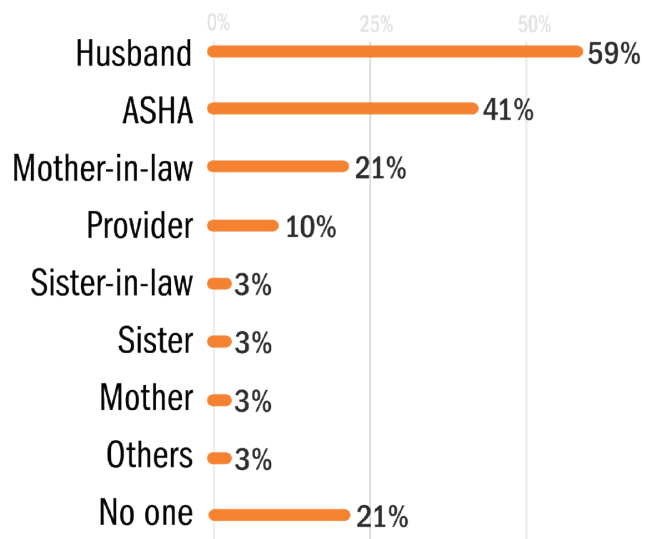

Multiple responses were possible

health worker to discuss stopping the method, and 62 percent returned to the same facility where they received the IUD.

Women who spoke to their husband about the desire to discontinue $(n=40)$ reported that husbands provided a range of advice. Seventy-three percent of women said their husband asked them to stop using the IUD, thirteen percent said their husband suggested switching to another method, and ten percent said their husband asked them to visit a doctor. Five percent suggested something else (data not shown).

Of those who spoke to an ASHA about their desire to stop using the IUD $(n=28)$, ten women reported that the ASHA asked them to stop using the method, six reported the ASHA accompanied them to the health facility, and five reported the ASHA provided counseling on other methods. Three reported receiving medicine for side effects from the ASHA, and two reported that the ASHA recommended they keep using the IUD. One reported the ASHA asked her to switch to another method and one reported something else (data not shown).

Figure 8 presents the proportion of interval IUD discontinuers who switched to a different modern method of contraception by whom they spoke to about their desire to discontinue the method. Those who spoke only to an ASHA were most likely to switch to another modern method (60\%), followed by those who spoke to neither their husband nor an ASHA (39\%) and those who spoke to both their husband and an ASHA (28\%). Discontinuers who spoke to their husband only were the least likely to switch to another modern method (14\%).
Figure 8

Percent of interval IUD discontinuers who switched to a modern method, by whom they spoke to

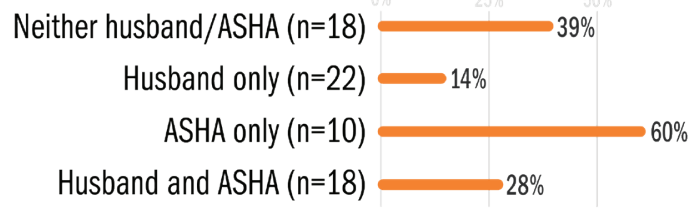

\section{DISCUSSION}

The IUD is an important long-acting, reversible contraceptive method, and an alternative to permanent female sterilization that does not carry the risk of regret. In India, as the government aims to expand the basket of reversible method choice available to women, understanding the experiences of those who use the interval IUD is paramount. Interval IUD users enrolled in this study were largely between the ages of 20 and 29, had attended secondary school or higher, had one or two children, and did not want to have any children in the future. Most were involved in the final decision to use the IUD and had met with a frontline health worker in the three months preceding the enrollment survey.

While some items of reported process quality at the time of interval IUD insertion were high, overall there is room to improve the quality of services clients receive. For example, less than half of the interval IUD users reported that they received information on potential side effects of the IUD and management of side effects. Since more than one third of the discontinuers cited side effects and health concerns as reasons for discontinuing, it is important that women who begin a new episode of interval IUD use do so with a robust understanding of what to expect regarding side effects and how to manage them.

Interval IUD users' responses may have recall bias, but this is likely to be minimal as they were interviewed within one month of the visit during which they had their IUD inserted. Additionally, many interval IUD users had received information from a frontline health worker about contraceptive methods in the three months preceding the enrollment survey; responses about quality of care at initiation of IUD may not have included this prior counseling.

Three months after beginning an episode of interval IUD use, 87 percent of respondents who participated in the follow up survey were still using the IUD. More than 70 percent of the discontinuers switched to another method, although less than half of those 
switched to another modern method; more switched to a traditional method. Those who discussed their desire to stop using with an ASHA only were more likely to switch to another modern method than those who spoke to their husband only, an ASHA and their husband, or neither. However, only 40 percent of respondents did speak with an ASHA about their desire to stop using the IUD, and only 18 percent of those who spoke to ASHAs were told to switch to other methods. Thus, it is important to reinforce with ASHAs the need to follow-up with new IUD users and discuss concerns about the method and the possibility of switching to another modern method, as long as the woman desires to prevent pregnancy.
More than half of the interval IUD users enrolled in the study reported their husbands were involved in the final decision to use the interval IUD, and most of the women who had discontinued use of the IUD by month three reported consulting their husbands about the desire to discontinue use. Given the high involvement of husbands in decision making regarding IUD use and discontinuation, it is important to ensure husbands are informed about possible side effects of the IUD, how to manage side effects, and the possibility of switching to another modern method as long as the desire to prevent pregnancy remains.

\section{REFERENCES}

International Institute for Population Sciences (IIPS) and ICF. 2017a. National Family Health Survey (NFHS-4), 2015-16. Mumbai, India.

International Institute for Population Sciences (IIPS) and ICF. 2017b. National Family Health Survey (NFHS-4), 2015-16 [Dataset]. IAIR73FL.DTA. Mumbai, India.

Jain A, Townsend J \& Ramarao S. 2018. Proposed metrics to measure quality: Overview. Population Council, NY.

Khan ME, Kar SS, Desai VK, Patel, P. 2007. Repositioning of IUD Program in Gujarat: Findings from Baseline Survey. Research Update No. 11. Population Council, New Delhi.

Ministry of Health and Family Welfare. 2013. IUCD Reference Manual for Medical Officers and Nursing Personnel. New Delhi, India.
Ministry of Health and Family Welfare. 2014. India's 'Vision FP 2020'. New Delhi, India.

National Health Mission. 2017. Family Planning: Background. Ministry of Health and Family Welfare, Government of India, New Delhi.

Scott K, Javadi D \& Gergen J. 2014. India's Auxiliary Nurse-Midwife, Anganwadi Worker, Accredited Social Health Activist, Multipurpose Worker, and Lady Health Visitor Programs. CHW Central.

World Health Organization. 2005. Report of a WHO Technical Consultation on Birth Spacing. Geneva, Switzerland.

\section{THE EVIDENCE PROJECT}

Population Council

4301 Connecticut Ave. NW

Washington, DC 20008

tel: +202 2379400

evidenceproject@popcouncil.org
(S) USAID

The Evidence Project is made possible by the generous support of the American people through the United States Agency for International Development (USAID) under the terms of cooperative agreement no. AIDOAA-A-13-00087. The contents of this document are the sole responsibility of the Evidence Project and Population Council and do not necessarily reflect the views of USAID or the United States Government.

Evidence

The Evidence Project uses implementation science-the strategic generation, translation, and use of evidence-to strengthen and scale up family planning and reproductive health programs to reduce unintended pregnancies worldwide. The Evidence Project is led by the Population Council in partnership with the Population Reference Bureau.

Suggested Citation: The Evidence Project. 2018. "Dynamics of interval IUD use in India: Quality of care received and interim results three months after insertion," Research Brief. Washington, DC: Population Council, The Evidence Project. 